\title{
Les récepteurs des FGF (fibroblast growth factor) et des virus herpétiques
}

Les FGF (fibroblast growth factors) sont des facteurs de croissance actifs sur la plupart des tissus d'origine mésodermique et neuroectodermique. Ils semblent avoir deux classes de récepteurs, à basse et à haute affinité, dont les $\mathrm{ADNc}$ ont été clonés. Les récepteurs à basse affinité, dont il existe des formes membranaires et, probablement, extracellulaires, fixent les FGF par l'intermédiaire d'une copule glucidique de type protéo-héparane sulfate alors que les récepteurs à haute affinité appartiennent à la famille des tyrosine kinases. Les virus herpès peuvent se fixer aux deux classes de récepteurs et être internalisés par endocytose du complexe virus/récepteur à haute affinité. La fixation du virus sur ce récepteur pourrait mimer la stimulation par le facteur de croissance et donc expliquer certains des effets mitogènes des virus herpès.

\section{Yves Courtois}

\section{ADRESSE}

Y. Courtois : directeur de recherches à l'Inserm. Unité de recherches gérontologiques, Inserm U. 118, 29 rue Wilhem, 75016 Paris,

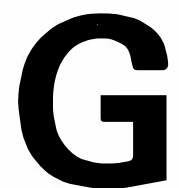

râce notamment aux travaux précurseurs de Stanley Cohen (prix Nobel de médecine, 1986) sur le facteur de croissance de l'épiderme (ou epidermal growth factor, EGF en anglais), on sait qu'un certain nombre de protéines de petite taille jouent un rôle dans la communication entre les cellules ou les organes et contrôlent de multiples étapes du développement embryonnaire, de la différenciation des tissus, de leur fonctionnement normal et même de leur sénescence. Ces protéines appelées facteurs de croissance parce qu'elles ont été isolées grâce à leur aptitude à faire proliférer in vitro les cellules sont donc au centre d'une intense activité de recherche scientifique et industrielle, non seulement pour leur rôle fondamental, mais aussi parce que de plus en plus on leur découvre des débouchés thérapeutiques potentiels intéressants, en particulier dans le domaine de la cicatrisation de la peau, de la cornée ou des régénérations osseuses et nerveuses. En outre il ne faut pas oublier que certains de ces facteurs pourraient être aussi impliqués dans des processus de transformation ou de cancérisation.

\section{Les récepteurs des FGF}

Or, l'activité biologique de ces facteurs dépend principalement du tissu cible qu'ils stimulent et de la présence à la surface cellulaire de récepteurs spécifiques. Certains de ces récepteurs ont été bien identifiés et leur isolement et clonage ont permis de montrer que leur activation par la fixation du facteur de croissance 
induisait des cascades de réaction dont la première semblait être l'autophosphorylation du récepteur luimême au niveau de ses résidus tyrosyl. Ce type de réaction place ces récepteurs dans la famille des tyrosines kinases qui comprend les récepteurs du facteur de croissance dérivés des plaquettes (platelet derived growth factor, PDGF), de l'EGF et du facteur de croissance des fibroblastes (fibroblast growth factor, FGF en anglais).

C'est le ou les récepteur(s) au FGF qui vient de faire l'objet d'une importante découverte qu'il convient de replacer dans son contexte. En effet, les FGF font partie d'une grande famille qui comprend au moins sept membres possédant de fortes analogies de structure (aFGF, bFGF, KGF, Int $\left.{ }^{2}, \mathrm{FGF}^{5}, \mathrm{FGF}^{6} \ldots\right)$. Comme leur nom ne l'indique pas, ils sont capables de stimuler la plupart des tissus d'origine mésodermique ou neuroectodermique et sont donc doués d'activités biologiques multiples. A titre d'exemple, ils induisent la différenciation des cellules musculaires dans les premières étapes de l'embryogenèse, ils peuvent stimuler la prolifération des cellules endothéliales vasculaires, ils prolongent la survie des neurones in vitro et in vivo, etc. Des méthodes indirectes ont permis d'identifier deux types de récepteurs de FGF, les uns ayant une faible affinité et les autres une forte affinité pour leur ligand. Les récepteurs de faible affinité sont présents en grande quantité dans les membranes basales séparant les tissus ainsi que dans celles des vaisseaux, de la cornée et de la capsule du cristallin. La spécificité de la liaison des FGF à ces récepteurs est assurée par la partie polysaccharidique d'un type spécial de protéoglycanes, les protéohéparane sulfates. En fait, l'une des propriétés les plus extraordinaires des FGF, propriété qui a été à l'origine de leur purification à homogénéité, est leur grande avidité pour l'héparine et les motifs " héparine-like " des protéohéparane sulfates. Notre équipe, en particulier, avait défini la nature de la liaison de ces facteurs sur les protéohéparane sulfates des membranes basales de l'œil [1] ou d'une tumeur de souris très riche en ces composants [2]. Un nouveau pas

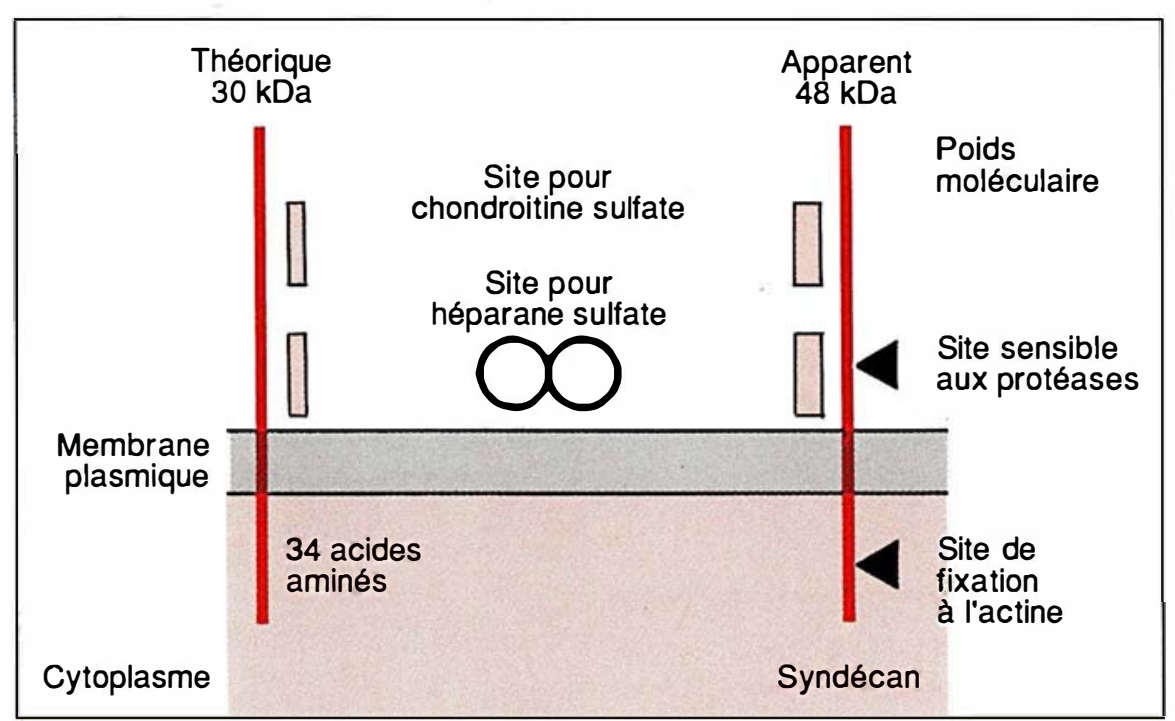

Figure 1. Représentation schématique des récepteurs de basse affinité pour le bFGF. A gauche le récepteur transmembranaire récemment cloné [3], à droite la syndécan [4]. Sur cette dernière, sur le corps protéique il existe des sites sur lesquels les chaînes de protéo-héparane sulfates peuvent se fixer. II est probable que des formes modifiées soient exprimées dans différents tissus.

vient d'être franchi par une équipe qui a récemment isolé un protéohéparane sulfate responsable de cette fixation [3] (figure 1). La méthodologie utilisée est exemplaire. Une librairie d'ADNc a été construite à partir de cellules de hamster (BHK 21) riches en récepteurs des FGF. Des cellules humaines lymphoïdes dépourvues de ces récepteurs ont été transfectées avec ces ADNc clonés. Les clones qui exprimaient les protéines fonctionnelles capables de fixer les FGF ont été isolés par leurs propriétés à se fixer sur des boîtes recouvertes de FGF (méthode du panning). Les séquences des $\mathrm{ADNc}$ responsables de cette fixation ont été déterminées; ils codent pour une protéine transmembranaire ayant un domaine extra-cellulaire possédant des sites de fixation pour les protéoglycanes et un domaine cytoplasmique homologue à des séquences capables de se lier à l'actine. Un protéoglycane analogue venait d'être découvert sous le nom de syndecan [4] chez la souris. Il semble donc que ces récepteurs (il y aurait plusieurs protéines ou gènes de la même famille) pourraient être les molécules de la membrane plasmique qui fixent les FGF. Comme ils ont un peptide signal, ils pourraient aussi être excrétés. En outre, ils possèdent une zone sensible aux protéases, au niveau de leur insertion dans la membrane et pourraient donc aussi servir de transporteurs ou de régulateurs de l'activité des FGF à distance. Participentils également au transport des FGF de l'intérieur vers l'extérieur de la cellule ? C'est une hypothèse intéressante qui, si elle était confirmée, résoudrait l'énigme de la sécrétion des FGF acide (aFGF) et basique (bFGF) qui n'ont pas les séquences classiquement retrouvées dans les peptides sécrétés. L'efficacité d'un transport des FGF sous une forme associée à des protéohéparane sulfates transmembranaires peut être évaluée par cotransfection de cellules qui ne produisent pas de FGF et n'ont pas de récepteurs avec les deux ADNc correspondants. Gageons que de telles expériences sont actuellement en cours de réalisation!

D'autres analogies de structure entre la syndecan et le récepteur de l'insu- 


\section{RÉFÉRENCES}

1. Jeanny JC, Fayein N, Moenner $M$, Chevallier B, Barritault D, Courtois Y. Specific fixation of bovine brain and retinal acidic and basic fibroblast growth factors to mouse embryonic eye basement membranes. Exp Cell Res 1987 : 171: 63-75.

2. Vigny M, Ollier-Hartmann MP, Lavigne $M$, et al. Specific binding of basic fibroblast growth factor to basement membrane like structures and to purified heparan sulfate proteoglycan of EHS tumor. I Cell Physiol 1988 ; 137 : 321-8.

3. Kiefer MC, Stephans JC, Crawford K, Okino K, Barr PJ. Ligand affinity cloning and structure of a heparan sulfate proteoglycan receptor for fibroblast growth factor. Science 1990, in press.

4. Saunders S, Jalkanen M, O'Farrell S, Bernfield M. Molecular cloning of syndecan, an integral membrane proteoglycan. $J$ Cell Biol 1989 ; 108 : 1547-56.

5. Imamura $T$, Tokita $Y$, Mitsui Y. Purification of basic FGF receptors from rat brain. Biochem Biophys Res Comm 1988 ; 155 : 583-90.

6. Lee PL, Johnson DE, Cousens LS, Fried VA, Williams LT. Purification and complementary DNA cloning of a receptor for basic fibroblast growth factor. Science 1989, 245 : 57-60.

7. Pasquale $\mathrm{HB}$, Singer SJ. Identification of a developmentally regulated proteintyrosine kinase by using antiphosphotyrosine antibodies to screen a cDNA expression library. Proc Natl Acad Sci USA 1989 ; 66 : 5449-53

8. Ruta M, Burgess W, Givol D, et al. Receptor for acidic fibroblast growth factor is related to the tyrosine kinase encoded by the fms-like gene (FLG). Proc Natl Acad Sci USA 1989 ; 86 : 8722-6.

9. Blanquet P, Patte C, Fayein N, Courtois Y. Identification and isolation from bovine epithelial lens of two basic fibroblast growth factor receptors that possess bFGF enhanced phosphorylation activities. Biochem Biophys Res Comm 1989 ; 160 : 1124-231. line font penser que ces protéines servent bien à la fois de liens entre les cellules et la matrice extracellulaire et de "fixateur" des facteurs de croissance.

Cette richesse des processus de liaison des FGF aux récepteurs de basse affinité se retrouve également pour la famille des récepteurs de haute affinité. C'est par des méthodes biochimiques [5] que furent obtenues les premières séquences protéiques des récepteurs de cette famille. Puis une utilisation judicieuse de techniques de biologie moléculaire, directes ou indirectes [6-8] (figure 2), permit d'en cloner les ADNc et les gènes. Comme plusieurs équipes [9] avaient suggéré que les récepteurs possédaient une activité tyrosine kinase et que les motifs responsables de cette activité enzymatique étaient très conservés, il a été possible d'isoler, à partir de banques $\mathrm{ADNc}$ ou de banques génomiques, des séquences nucléotidiques qui codent probablement pour des récepteurs des FGF. C'est ce qui a permis à l'équipe de Schlessinger de mettre en évidence un récepteur humain (Flg) [8]. Récemment l'équipe de R. Breathnach (Nantes) avec notre collaboration a isolé un autre récepteur humain TK 14 qui est différent de Flg [10]. Une autre approche moins classique a été celle de l'équipe de Pasquale et Singer [7]. Partant de l'observation que des bactéries infectées par des phages n'expriment pratiquement pas d'activité tyrosine kinase, ils ont infecté des bactéries par des phages portant les informations provenant de banques d'ADNc d'embryons de poulet. Grâce à des anticorps antiphosphotyrosine, ils mirent en évidence des clones ayant des activités tyrosine kinases liées à l'expression des ADNc de poulet. De cette façon, un, puis plusieurs ADNc codant pour des protéines tyrosine kinases ont été isolés, séquencés

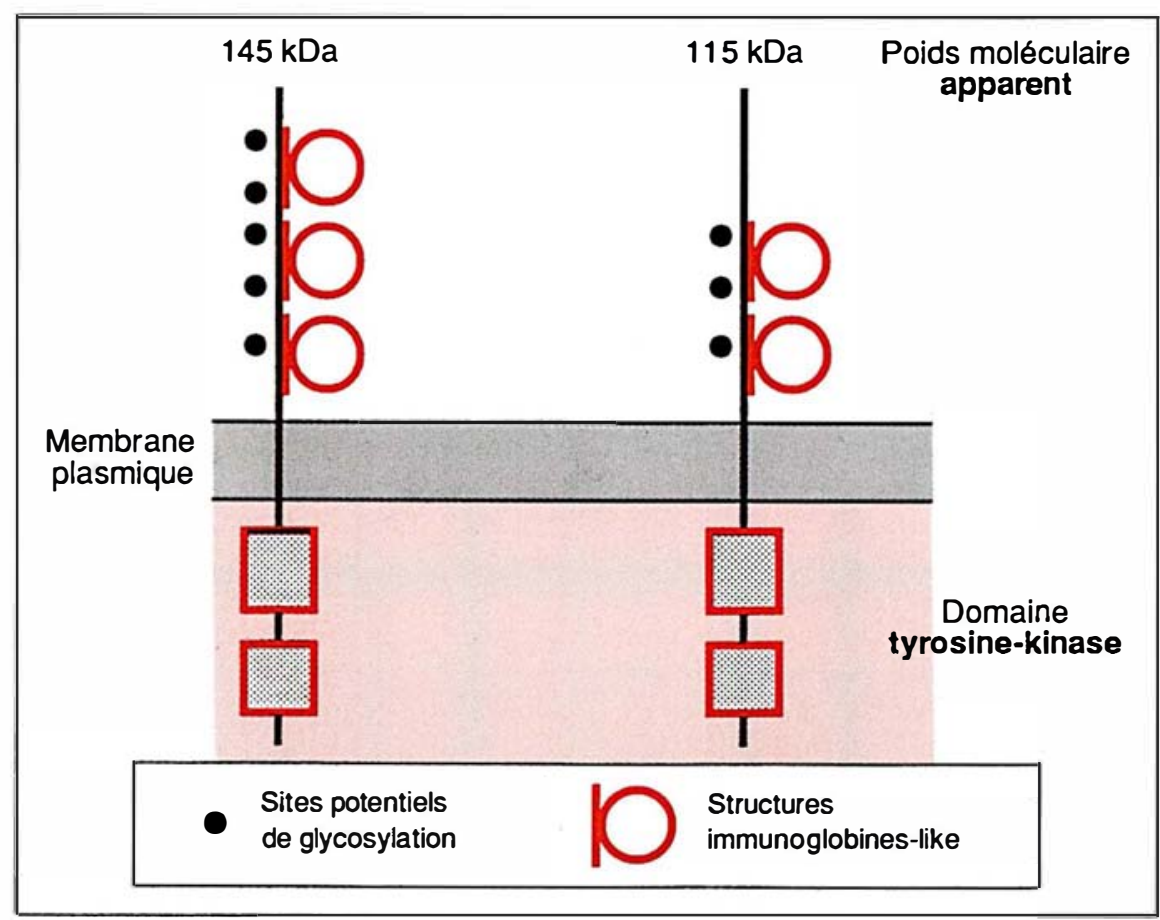

Figure 2. Représentation schématique des récepteurs de haute affinité pour les FGF. La partie extracellulaire possède de nombreux sites potentiels de glycosylation, nécessaires pour leur activité biologique. Les nombreuses cystéines pourraient former des ponts $S$-S et créer des structures en boucle. Actuellement on peut distinguer deux classes de récepteurs avec des corps protéiques différents pouvant avoir deux ou trois domaines immunoglobulines $[6-8,10,17,18]$.

$\mathrm{m} / \mathrm{s} n^{\circ} 7 \mathrm{vol} .6$, septembre 90 


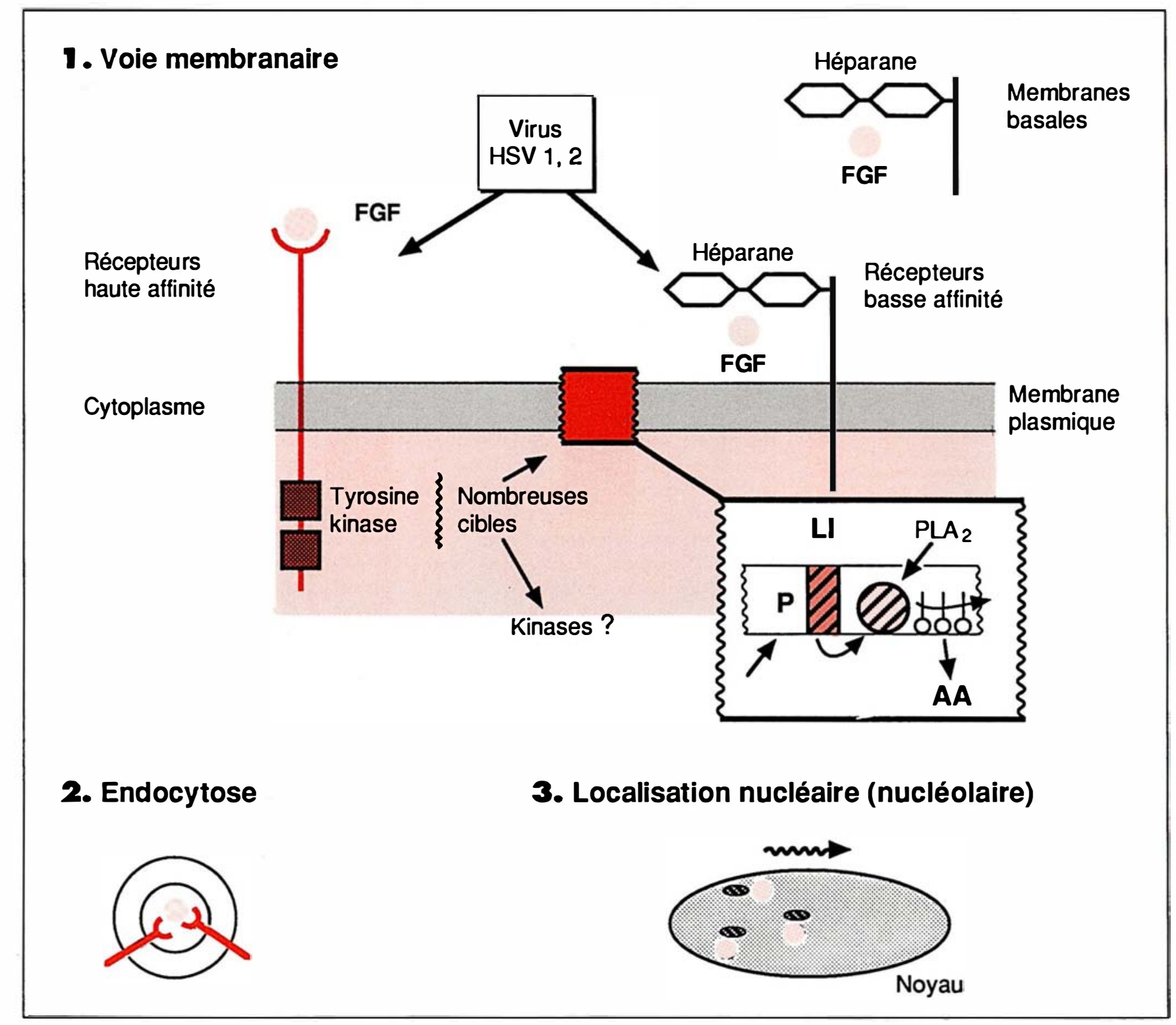

Figure 3. Mécanisme d'action des facteurs de croissance des fibroblastes sur une cellule. Le FGF $1 O$ I se fixe soit sur le récepteur à haute affinité, soit sur un récepteur de basse affinité. Dans le premier cas, il déclenche une activité tyrosine kinase, se phosphorylant lui-même et phosphorylant d'autres cibles. Parmi celle-ci la lipocortine (LI), une protéine membranaire qui active la phospholipase $A_{2}\left(P L A_{2}\right)$ provoquant la formation d'acide arachidonique (AA). Le récepteur subit alors une endocytose avec le ligand et ce dernier est dégradé. Par un mécanisme encore inconnu dans certains cas [14], le bFGF est retrouvé dans le noyau au niveau des nucléoles. II active alors la transcription de l'ARN ribosomal. Le bFGF a aussi la possibilité de se fixer sur un protéoglycan transmembranaire, le récepteur de basse affinité, probablement sur les chaînes héparane-sulfate. On ne sait pas si ce mode de fixation transduit un message à la cellule par l'intermédiaire de son domaine intracellulaire. Le protéo-héparane sulfate pourrait aussi stocker le FGF jusque dans les membranes basales, le protégeant ainsi de la dégradation par des enzymes protéolytiques. Le virus de I'Herpès (HSV-1-HSV-2) utiliserait le récepteur de haute affinité pour pénétrer dans la cellule mais pourrait aussi être stocké par les récepteurs de basse affinité que ce soit par une interaction directe ou par l'intermédiaire d'un complexe avec le FGF. 


\section{RÉFÉRENCES}

10. Houssaint E, Blanquet PR, ChampionArnaud P, Gesnel MC, Courtois Y, Breathnach R., Proc Natl Acad Sci USA 1990 ; in press.

11. Fayein N, Courtois Y, Jeanny JC. Ontogeny of basic fibroblast growth factor binding sites in mouse ocular tissues during embryonic and post-natal development. Exp Cell Res 1990 ; 188 : 75-88.

12. Magnaldo I, L'Allemain G, Chambard JC, Moenner M, Barritault D, Pouysségur J. J Biol Chem 1986; 261 : 16916-22.

13. Blanquet $\mathrm{P}$, Paillard $\mathrm{S}$, Courtois $\mathrm{Y}$. Phosphorylation and phosphorylase A 2 activity of a $34 \mathrm{Kd}$ surface protein in lens epithelial cells : relation to mitogenic signal induced by basic fibroblast growth factor. Growth Factors $1990 ; 3: 15-24$.

14. Baldin V, Roman AM, Bosc-Bierne I, Amalric $F$, Bouche $G$. Translocation of bFGF to the nucleus is $\mathrm{G} 1$ phase cell cycle specific in bovine aortic endothelial cells. Embo J 1990 ; 9 : 1511-7.

15. Kaner RJ, Baird A, Mansukhani A, et al. Fibroblast Growth Factor receptor is a portal of cellular entry for Herpes simplex virus type I. Science 1990 ; in press.

16. Stein CA, La Rocca RV, Thomas R. Suramin : an anticancer drug with a unique mechanism of action. J Clin Oncol $1989 ; 7$ : 499-508.

17. Reid H, Wilks A, Bernard O. Two forms of the basic fibroblast growth factor receptor-like mRNA are expressed in the developing mouse brain. Proc Natl Acad Sci USA 1990 ; 87 : 1596-600.

18. Olwin B, Burnus L, Kudha A, Lueddecke B, Shaw D, Zuber M. Characterization of a putative fibroblast growth factor receptor for acidic and basic fibroblast growth factors. J Cell Biochem 1990 ; 14F : puis comparés à ceux codant pour les récepteurs humains des FGF. A ce jour, il apparaît clairement qu'il existe dans chaque espèce des familles de récepteurs à haute affinité pour les FGF qui ont tous une structure assez proche, notamment dans la partie tyrosine kinase intracellulaire. Le domaine extracellulaire, pour sa part, comporte deux ou trois boucles qui lui donnent une structure de type immunoglobuline, structure que l'on retrouve dans d'autres récepteurs comme les intégrines. Cette structure, grâce à des branchements $\mathrm{N}$ glycosyles, permet la fixation des FGF.

Les divers membres de la famille des récepteurs ont-ils des affinités différentes vis-à-vis des FGFa ou b, ou des facteurs apparentés? Existe-t-il une expression différentielle de ces récepteurs dans le temps (au cours du développement, de la sénescence), suivant les tissus ou lors de l'apparition de processus pathologiques? Dans notre unité, l'équipe de J.C. Jeanny [11] a déjà montré que les récepteurs de haute affinité, mis en évidence par la fixation de FGF iodé, se condensaient dans les régions riches en synapses de la rétine neurale en cours de formation. Il est vraisemblable qu'une réponse à ces questions va maintenant pouvoir être apportée bientôt puisque les outils pour ce faire existent.

De même, à part l'activité tyrosine kinase qui semble être cruciale pour la transduction du message par le récepteur à l'intérieur de la cellule, on ne connaît pas non plus en détail les cibles de la cascade d'événements qui va déclencher la réponse de la cellule stimulée (figure 3, p. 677). Suivant les cellules, ces voies peuvent être différentes, comme l'a montré J. Pouysségur [12], éventuellement indépendantes de la voie d'activation de la phospholipase C. Dans notre unité, l'équipe de $\mathrm{P}$. Blanquet a étudié le mécanisme d'activation des cellules endothéliales vasculaires et épithéliales du cristallin mises en prẹsence de FGF [13]. Cette équipe a montré que les FGF modulent la phosphorylation d'une famille de protéines, appelées lipocortines, qui participent selon leur état de phosphorylation à l'activation ou à l'inhibition de la phospholipase A2, une enzyme membranaire. Ces facteurs de croissance contrôlent ainsi le métabolisme de l'acide arachidonique et de ses dérivés qui pourraient jouer un rôle important dans le contrôle du cycle cellulaire. Cependant, il y a d'autres possibilités de transduction du message qui restent à explorer (médecine/sciences, le transfert des signaux mitogéniques : une affaire de particules, O. Filhol, C. Cochet, sous presse). Ainsi dans des cellules endothéliales vasculaires synchronisées, l'équipe de G. Bouche [14] a montré que le bFGF est transporté dans le noyau de la cellule, pendant une période très courte précédant la stimulation de la synthèse de l'ADN, et qu'il active spécifiquement et directement la transcription des ADN ribosomaux et la phosphorylation d'une protéine nucléolaire, la nucléoline. Il y a donc peut-être plusieurs possibilités d'activation d'une cellule, les unes passant par l'activation de récepteurs membranaires, les autres par une activation nucléaire directe après internalisation du complexe ligand-récepteur. Là encore, tous les outils existent pour permettre de résoudre ces énigmes et comprendre enfin les mécanismes intimes de communication cellulaire à laquelle participent ces facteurs.

\section{Les virus de l'herpès et le FGF ou l'opportunisme des virus}

Une autre découverte récente va encore accélérer les recherches dans ces domaines. En effet, les deux grandes familles de récepteurs des FGF, celle de haute affinité et celle de basse affinité, sont très certainement impliquées dans la pénétration des virus de l'herpès (HSV-1 et HSV-2) dans les cellules. Cette découverte a été faite d'abord fortuitement par le groupe de Hajjar [15] qui avait remarqué que l'infectabilité de cellules musculaires traitées par le virus de l'herpès HSV-1 était considérablement diminuée par l'addition de FGF. Rapidement, cette observation fut affinée et généralisée grâce à des travaux menés en collaboration avec l'équipe de A. Baird [15] qui montrent clairement que le bFGF empêche spécifiquement la fixation 
du virus sur le récepteur du FGF; de plus, des peptides synthétiques représentant la fraction du FGF qui a de l'affinité pour son récepteur empêchent les cellules d'être infectées. Il s'agit bien d'un mécanisme passant par le récepteur puisque si, au lieu d'être traité en même temps par le bFGF (ou un analogue), la cellule est traitée par le bFGF après avoir été mise en présence du virus, il n'y a plus d'effet d'inhibition. Des expériences complémentaires effectuées en collaboration avec Basilico [15] ont montré que des cellules non infectables par ces virus n'ont pas de récepteurs à haute affinité au FGF, alors que transfectées avec des ADNc codant pour ces récepteurs, elles deviennent infectables. D'ailleurs, la fixation du virus entraînerait la phosphorylation du récepteur. Cela déclenche-t-il aussi la prolifération des cellules infectées ? Il est probable que la réponse à cette question, cruciale pour comprendre certains aspects de la pathogénicité du virus, ne va maintenant pas tarder à être donnée. Mais ce qui rend encore plus passionnant ce parallélisme entre FGF et les virus HSV-1 c'est que ces virus utilisent probablement aussi les sites de basse affinité du FGF pour se fixer sur la cellule. On savait déjà que les virus HSV-1 et -2 s'attachaient initialement à la surface des cellules sur des protéoglycanes par l'intermédiaire de glycoprotéines de l'enveloppe virale $\mathrm{gB}$ et $\mathrm{gD}$. L'équipe de Kiefer cite des expériences dans lesquelles ils inhibent, en utilisant les glycoprotéines $\mathrm{gB}$ et $\mathrm{gD}$, la fixation de cellules transfectées par l'ADNc du récepteur FGF héparane sulfate de basse affinité à des boîtes couvertes de FGF avec les glycoprotéines $\mathrm{gB}$ et $\mathrm{gD}$. L'héparine et les polyanions sont aussi connus pour empêcher l'infection de certaines cellules par HSV-1.

Toutes ces découvertes sont terriblement stimulantes. Elles nous rappellent d'abord que les virus HSV ont développé des stratégies de pénétration dans la cellule qui sont utilisées normalement par la cellule pour d'autres fonctions biologiques. Les chercheurs sont à chaque fois étonnés, mais c'est cette "stratégie du coucou " qui est utilisée par d'autres virus comme le HIV, virus du $\mathrm{m} / \mathrm{s} n^{\circ} 7 \mathrm{vol}$. 6, seplembre 90
SIDA, qui utilise un récepteur glycoprotéique CD4, comme le rhinovirus, qui utilise une protéine d'adhérence ICAM-1 pour être véhiculé dans la cellule, comme le virus de la rage qui utilise le récepteur à l'acétylcholine, comme le réovirus qui utilise le récepteur $\beta$-adrénergique, et enfin comme le virus de la vaccine qui interagit avec le récepteur de l'EGF $\left(\mathrm{m} / \mathrm{s} n^{\circ} 6\right.$, vol. 5 , p. $431 ; n^{\circ} 4$, vol. 6, p. $403 ; n^{\circ} 5$, vol. 6 , p. 485)

Le virus de HSV-1 va peut-être plus loin puisqu'il semble utiliser les deux types de récepteurs du FGF, ceux de haute et de basse affinité. Peut-être parce que ceux-ci coopèrent de toute façon, même pour la stimulation des cellules par le bFGF, ou qu'ils représentent des entités indépendantes. On pourrait aussi imaginer que les protéo-héparane sulfates représentent des réservoirs de stockage des bFGF et des virus herpès qui les présentent alors au récepteur de haute affinité. Quoi qu'il en soit, ces découvertes ouvrent des voies d'approche tout à fait nouvelles pour lutter contre ces virus, avec la fabrication d'antagonistes qui pourront, soit empêcher la fixation des virus sur les sites de basse affinité (par exemple des analogues de l'héparine dépourvus d'activité thrombolytique, des oligosaccharides synthétiques ou des analogues du bFGF), soit des peptides synthétiques ou des analogues bloquant la fixation sur les sites de haute affinité et ainsi la pénétration du virus. De tels analogues pourront être conçus grâce aux recherches qui se développent sur les relations structure-activité des facteurs de croissance et de leurs récepteurs. Probablement une approche mixte permettant de masquer ces deux classes de récepteurs sera-t-elle la plus efficace. Mais il ne faut pas oublier que les récepteurs des FGF sont très ubiquitaires et que les stratégies nouvelles devront tenir compte de ces faits pour éviter des effets secondaires indésirables. Par exemple, des produits [16] comme la suramine ont la propriété de désactiver les récepteurs au facteur de croissance, ce qui est potentiellement intéressant dans le traitement de certains cancers, mais elles ont aussi des effets secondaires (démyélinisation des nerfs périphériques) qui résultent peut-être de leur action sur les FGF impliqués dans le fonctionnement normal du système nerveux.

Les implications industrielles mondiales de ces recherches n'échappent à personne ; espérons qu'en France et en Europe, des équipes nouvelles viendront se joindre à celles modestement impliquées dans cette aventure, pour ne pas rester sur le quai du train en marche

\section{Summary}

Fibroblast growth factor and Herpes simplex virus receptors

FGF (fibroblast growth factors) are polypeptides very active on cells from mesodermal or ectodermal origin. They use two different classes of receptors whose cDNAs have been cloned. The low affinity receptors are transmembrane proteins, and possibly extracellular derivatives bind $\mathrm{FGF}^{\mathrm{F}}$ through their heparan sullate moiety, whilc high affinity receptors bclong to the tyrosine kinase family. Herpes virus HSV seems to bind to both FGF high affinity and low affinity receptors. Thus FGF receptors are the site of entry of HSV-1.

\section{Remerciements}

Je remercie particulièrement $P$. Blanquet et $G$ Fuhrmann pour les conseils qu'ils m'ont prodigués lors de la rédaction de cette revue.

\section{TIRÉS A PART}

Y. Courtois. 